\title{
Comparison of Cementless Calcar-Replacement Hemiarthroplasty With Proximal Femoral Nail for the Treatment of Unstable Intertrochanteric Fractures at Older Age Group
}

\author{
Anıl Agar ${ }^{1}$, Adem Sahin ${ }^{1}$, Orhan Gunes ${ }^{1}$, Deniz Gulabi ${ }^{1}$, Cemil Erturk ${ }^{1}$ \\ 1. Orthopaedics and Traumatology Department, Saglik Bilimleri University, Kanuni Sultan Suleyman Training and \\ Research Hospital, Istanbul, TUR
}

Corresponding author: Anıl Agar, anilagar@gmail.com

\begin{abstract}
Background: The aim of this study was to compare the outcomes of unstable intertrochanteric femur fractures treated with cementless calcar-replacement bipolar hemiarthroplasty $(\mathrm{CRH})$ and proximal femoral nail (PFN) in elderly patients.

Methods: All consecutive unstable intertrochanteric fractures treated with cementless CRH or PFN at our institution between January 2015 and January 2019 were reviewed retrospectively. The primary outcome measures were postoperative complications, reoperation rate, and hip function. The secondary outcome measures were intraoperative blood loss, transfusion rate, surgical time, hospital stay, and two- year mortality.
\end{abstract}

Results: Ninety-four patients in the hemiarthroplasty group and 77 patients in the PFN group were included for analysis. There were no significant differences between the two groups regarding the complications, ASA score, and reoperation rate. Significant differences were found between hemiarthroplasty and PFN group in comparison of the average length of hospital stay $(\mathrm{P}<0.05)$, time from hospitalization to operation $(\mathrm{P}<$ $0.05)$, intraoperative blood loss $(\mathrm{P}<0.001)$, transfusion rate $(\mathrm{P}<0.001)$, operation time $(\mathrm{P}<0.001)$, Harris Hip Score (HHS; $\mathrm{P}<0.001)$, and two-year mortality $(\mathrm{P}<0.05)$.

Conclusion: Both hemiarthroplasty and PFN produce satisfactory results in surgically treated unstable intertrochanteric femur fractures in the elderly. Both groups are associated with their own complications, but in the PFN group, better functional results, less surgery-related trauma, and lower mortality rates are the main advantages.

Review began 01/14/2021 Review ended 01/17/2021 Published 01/22/2021

\section{() Copyright 2021}

Agar et al. This is an open access article distributed under the terms of the Creative Commons Attribution License CC-BY 4.0., which permits unrestricted use, distribution, and reproduction in any medium, provided the original author and source are credited.
Categories: Orthopedics

Keywords: femur intertrochanteric fracture, calcar replacement, bipolar hemiarthroplasty, proximal femoral nail, harris hip score

\section{Introduction}

The incidence of intertrochanteric femoral fractures, which is generally seen in the elderly population and causes significant mortality and morbidity in orthopedic practice, is increasing due to prolonged life expectancy [1]. As patients age and bone quality decreases, unstable pattern fractures occur. Intertrochanteric femoral fractures constitute approximately $45-50 \%$ of hip fractures in the elderly population [2] and 50-60\% of these are of an unstable pattern [3]. The main goal of treatment of patients with this type of fracture is to return to their preoperative medical condition and daily activities [4]. Therefore, treatment aims for the patient to regain their pre-fracture level of activity by ensuring that the patient moves as soon as possible and to prevent the development of complications that may result in death due to immobility.

It is very important to provide effective and appropriate treatment in these fractures, which tend to be unstable due to decreased bone quality, especially due to the patient's age [5]. Many treatment methods such as proximal femoral nail (PFN), dynamic hip screw (DHS), external fixator, unipolar and bipolar hemiarthroplasty $(\mathrm{BPH})$ have been used in the treatment of these fractures [6,7]. However, it is very difficult to provide stable fixation in these unstable class fractures due to decreased bone quality.

The purpose of treatment is to restore ambulation safely and efficiently while minimizing the risk of medical complications and technical failure. Patients can return to their pre-injury levels earlier by being treated with any of these methods, thereby eliminating postoperative complications caused by prolonged immobilization or implant failures [8]. 
The primary aim of this study was to compare the functional results of patients who underwent cementless calcar-replacement hemiarthroplasty (CRH) and patients who underwent PFN due to unstable intertrochanteric fractures over 65 years of age. The secondary objective was to compare intraoperative and postoperative complications in both groups.

\section{Materials And Methods}

A retrospective examination was made of patients who were hospitalized and treated surgically for intertrochanteric femur fractures at the University of Health Sciences, Kanuni Sultan Süleyman Training and Research Hospital between January 2015 and January 2019. The patients included were those over 65 years of age with an unstable intertrochanteric hip fracture (according to Evans classification; types 3, 4, and 5). Exclusion criteria were a history of osteoarthritis in the hip joint, pathological fractures, bilateral fractures, age $<65$ years, treatment with a method other than cementless CRH or PFN, fractures that developed secondary to a tumor, Paget's disease, or metabolic bone disease, multiple trauma with head and/or chest trauma, additional fractures of the same lower extremity, rheumatic disease, and inadequate follow-up.

The clinical and radiographic features of the patients on first admission, surgery, and final follow-up were evaluated retrospectively. The collected data of the patients included demographic characteristics, time from injury to surgery, length of stay in the hospital, type of fracture according to AO/ASIF and Evans classification, American Society of Anesthesiologists (ASA) physical condition classification (ASA grade), surgical procedure type uncemented CRH (TST, Istanbul, Turkey) or PFN with osteosynthesis (TST, Istanbul, Turkey), operation time, bleeding amount, blood transfusion, and complications.

All operations were performed by the same experienced surgical team. Before the surgery, the surgeon who performed the operation gave information to the patient about both surgical methods and it was decided as a result of a joint decision on which procedure to be performed. Low molecular weight heparin was started as antithrombotic prophylaxis from the time of hospitalization to all patients and was continued for one month. Antibiotic prophylaxis of cefazolin sodium was administered 30 minutes preoperatively and was continued for 24 hours postoperatively. Anteroposterior and lateral radiographs were obtained 24-72 hours postoperatively and were analyzed for reduction and position of the implant.

All patients were encouraged to do active and passive functional exercise from postoperative day 1 under the supervision of an experienced physiotherapist. Patients who underwent CRH were permitted full weightbearing on the first postoperative day with the help of a walker. In the patient group applied with PFN, the patient was mobilized with partial weight-bearing on the postoperative first day with the help of a walker, and full weight-bearing was permitted at an average of 4 weeks according to the surgeon's decision in the follow-up examinations.

The patients were followed up every month for the first three months and then every three months for the first year and at six-month intervals thereafter. Complications were classified as problems not requiring revision surgery, such as bedsore, superficial infection, venous thromboembolism, and as problems requiring revision surgery, such as implant-related problems (cut-out, lateral sliding), deep infection, nonunion, hip dislocation, or secondary fracture.

The functional outcomes of the patients were evaluated with the Harris Hip Score (HHS) at the final followup examination by a physician blinded to the surgical technique [9]. The average of the scores obtained was used for statistical analysis. The degree of pain, performance of daily activities, and range of motion were evaluated.

\section{Statistical evaluation}

Data obtained in the study were analyzed statistically using IBM SPSS Statistics 22.0 software (IBM Corp., Armonk, New York). Conformity of the data to normal distribution was evaluated with the KolmogorovSmirnov test. Descriptive statistics were stated as mean \pm standard deviation (SD) values. The Student's ttest was used to compare quantitative data between two groups, and the Mann Whitney U test for two-group comparisons of non-normally distributed parameters. In the comparison of qualitative data, the Chi-Square test and the Continuity Correction (Yates) test were used. A value of $\mathrm{p}<0.05$ was accepted as statistically significant.

\section{Results}

A total of 626 intertrochanteric fracture operations were performed between 2015 and 2019. The study included 171 patients who met the inclusion criteria, comprising 94 patients in the CRH group and 77 patients in the PFN group (typical cases are presented in Figures 1-6). The CRH group included 27 (28.7\%) males and 67 (71.3\%) females with a mean age of 82.2 years (range, 71-94 years). The PFN group included 21 (27.3\%) males and 56 (72.6\%) females with a mean age of 81.5 years (range, 65-99 years). The average followup time was 23.3 months (range, 15-35 months) in the CRH group and 21.9 months (range, 13-28 months) in the PFN group. There was no significant difference between the CRH and PFN group in terms of demographic data (Table 1). 


\section{Cureus}

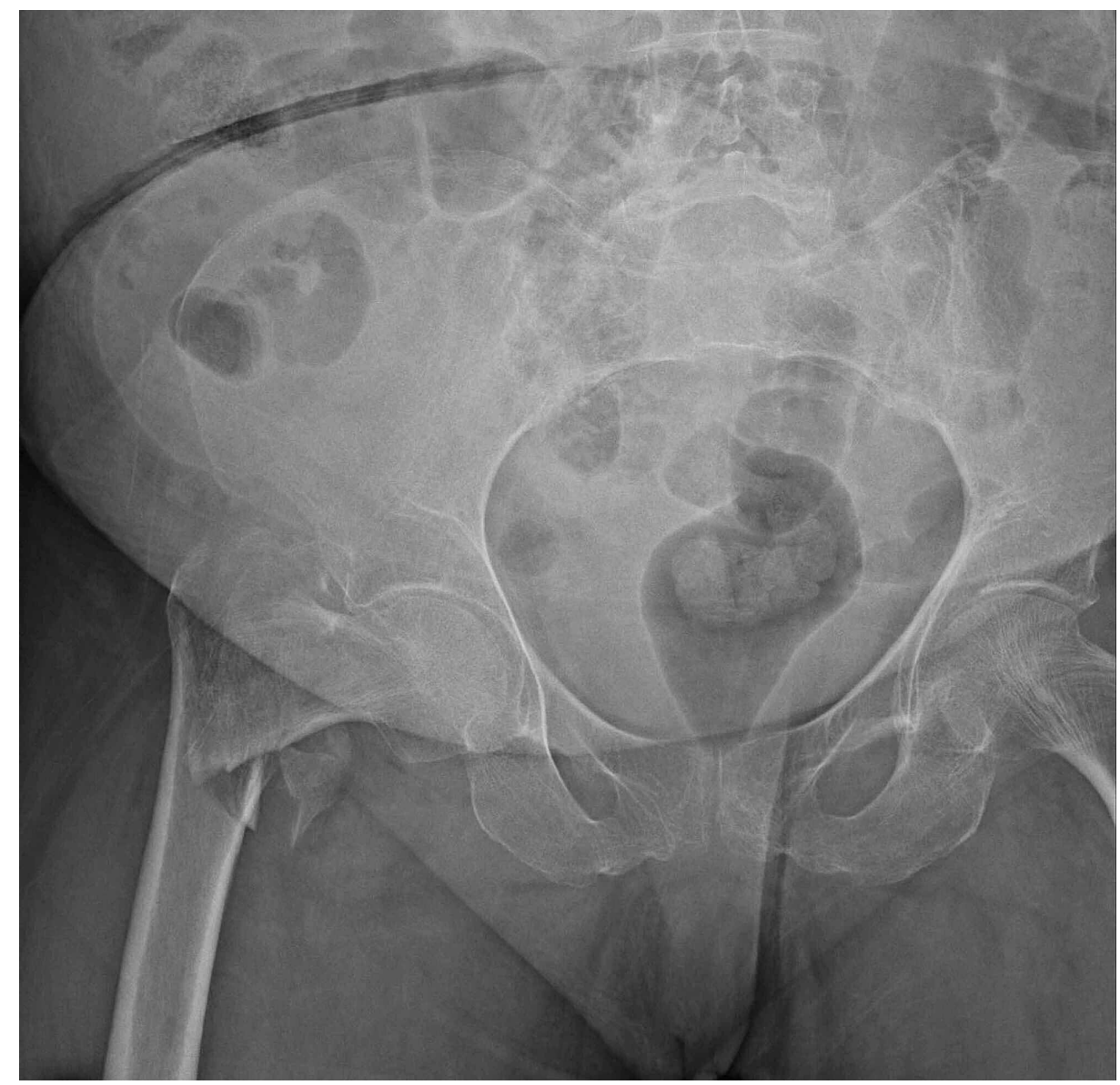

FIGURE 1: A 84-year-old female patient with a simple fall, right hip intertrochanteric fracture (type 5 according to Evans classification, type $A 2$ according to $A O$ classification). Cementless $\mathrm{CRH}$ applied to the patient.

$\mathrm{CRH}$ : calcar-replacement hemiarthroplasty. 


\section{Cureus}

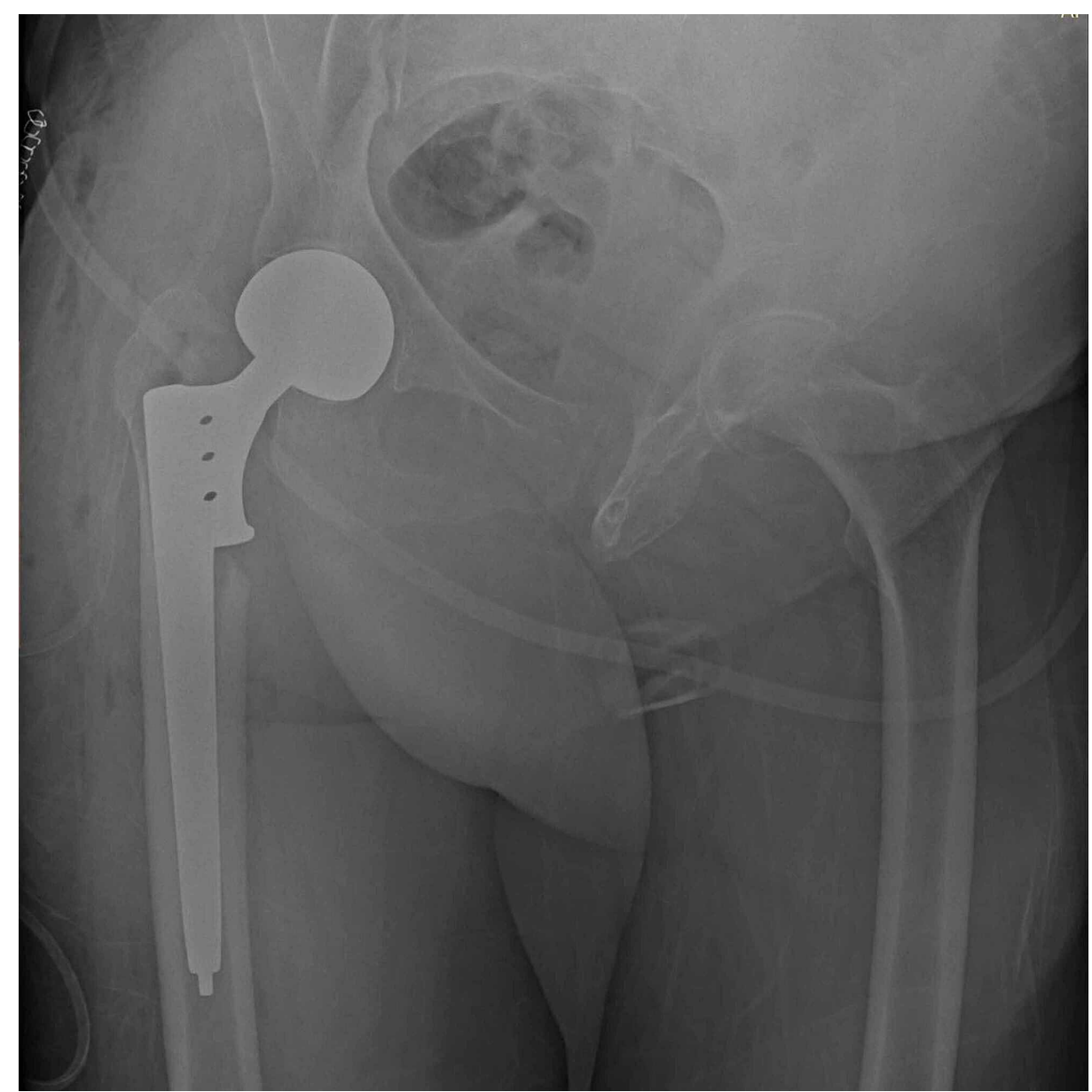

FIGURE 2: Postoperative first-day anterior-posterior hip radiography

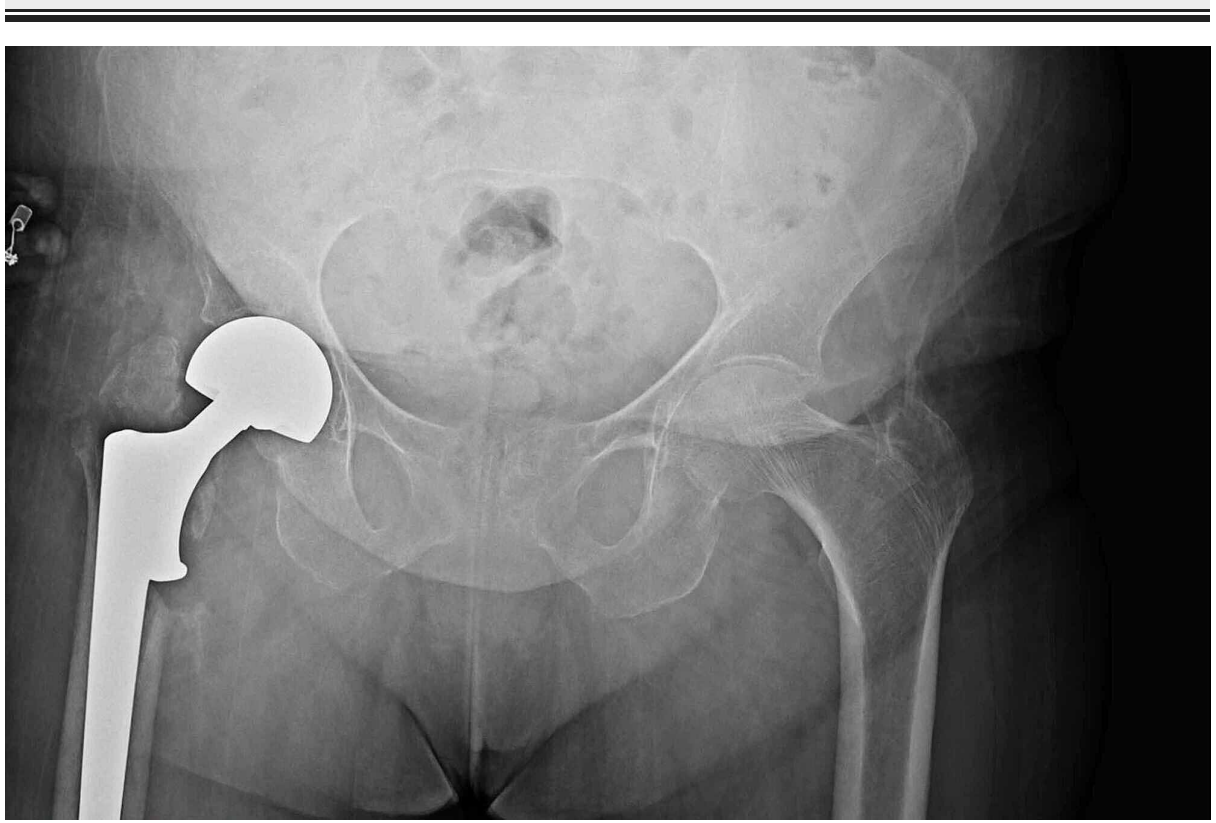

FIGURE 3: Anterior-posterior hip radiography at the third postoperative month of the patient 


\section{Cureus}

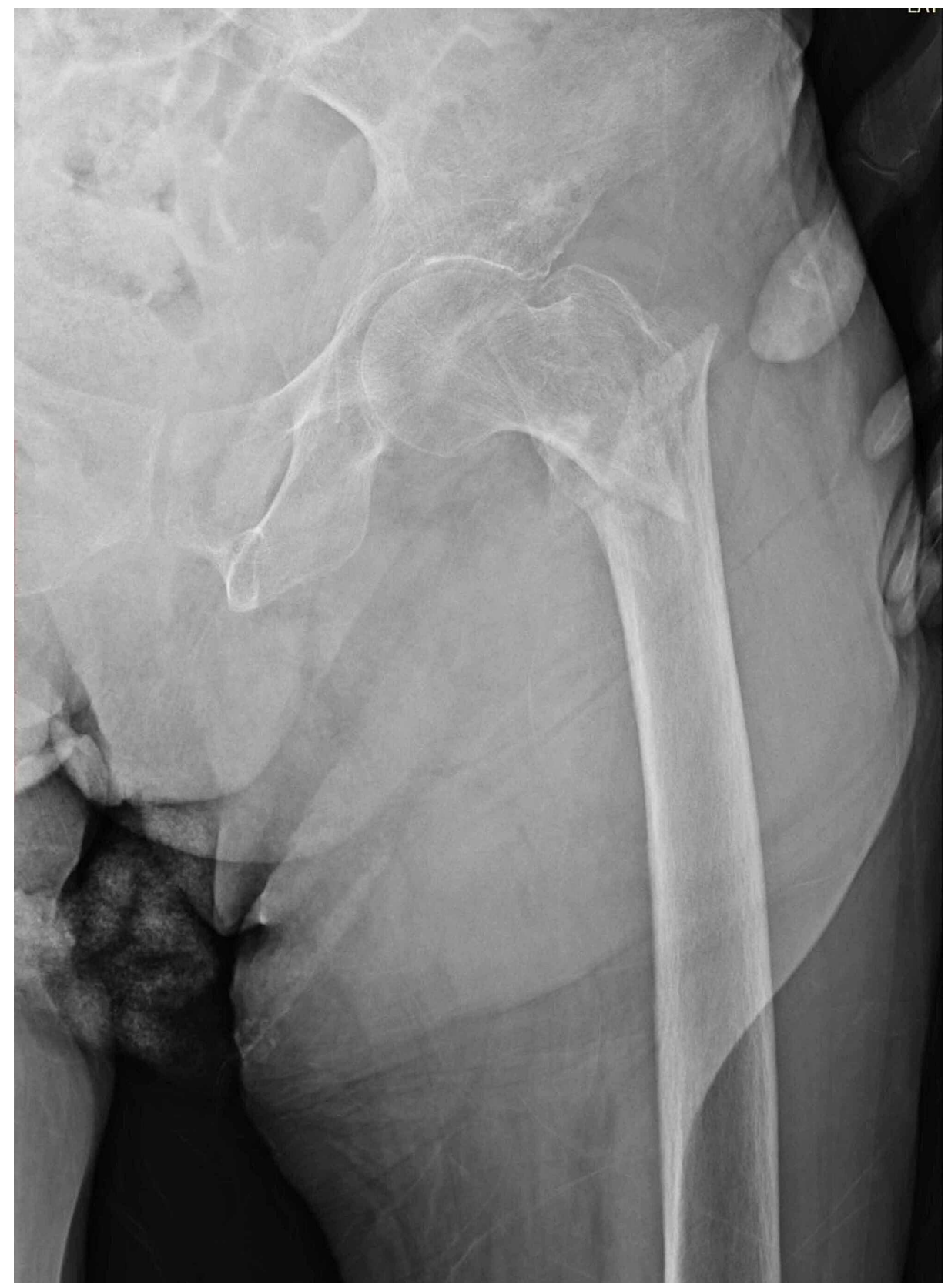

FIGURE 4: A 90-year-old female patient with a simple fall, left hip intertrochanteric fracture (type 4 according to Evans classification and type $\mathrm{A} 2$ according to $\mathrm{AO}$ classification). PFN applied to the patient.

PNF: proximal femoral nailing. 


\section{Cureus}

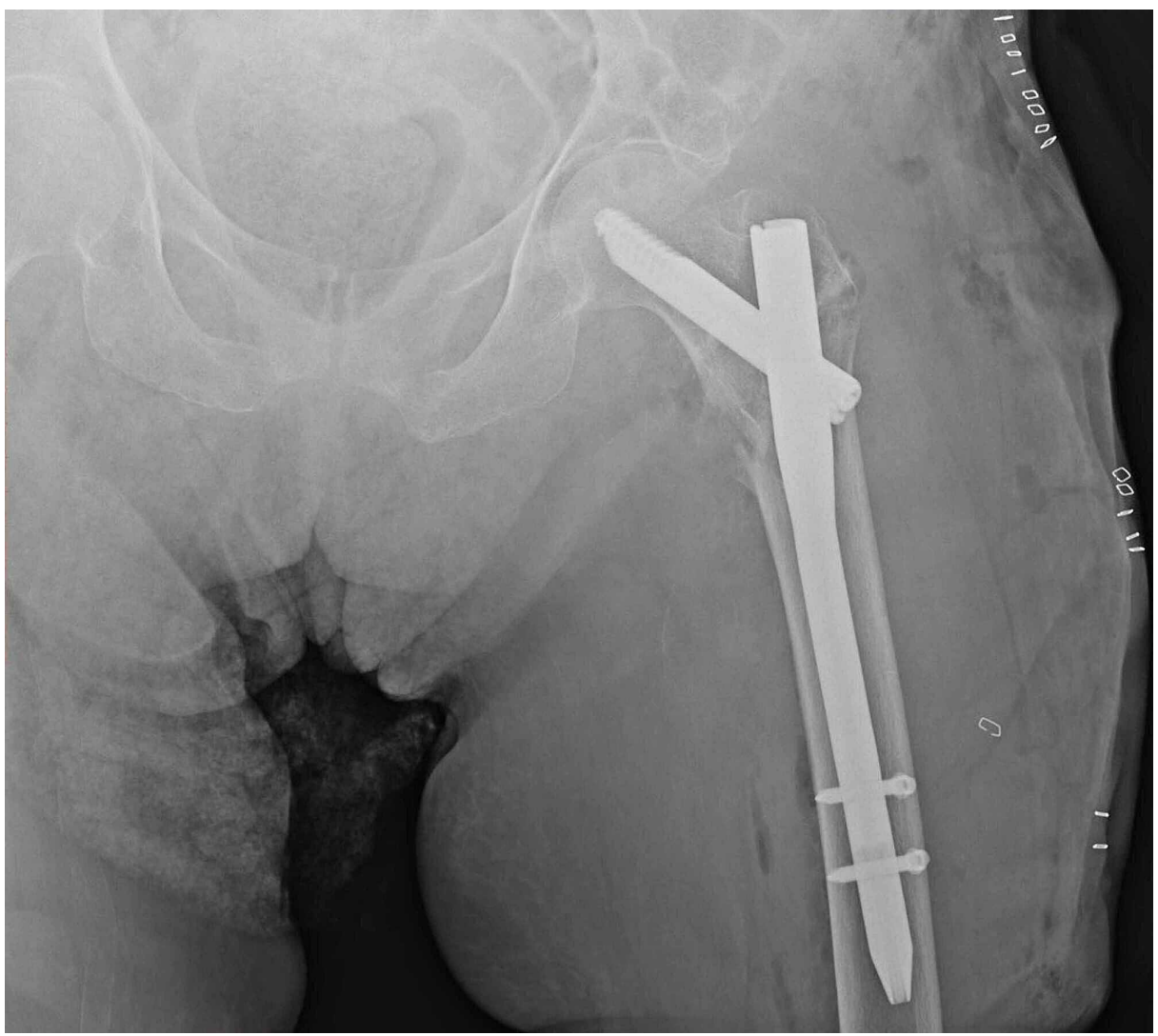

FIGURE 5: Postoperative first-day anterior-posterior hip radiography 


\section{Cureus}

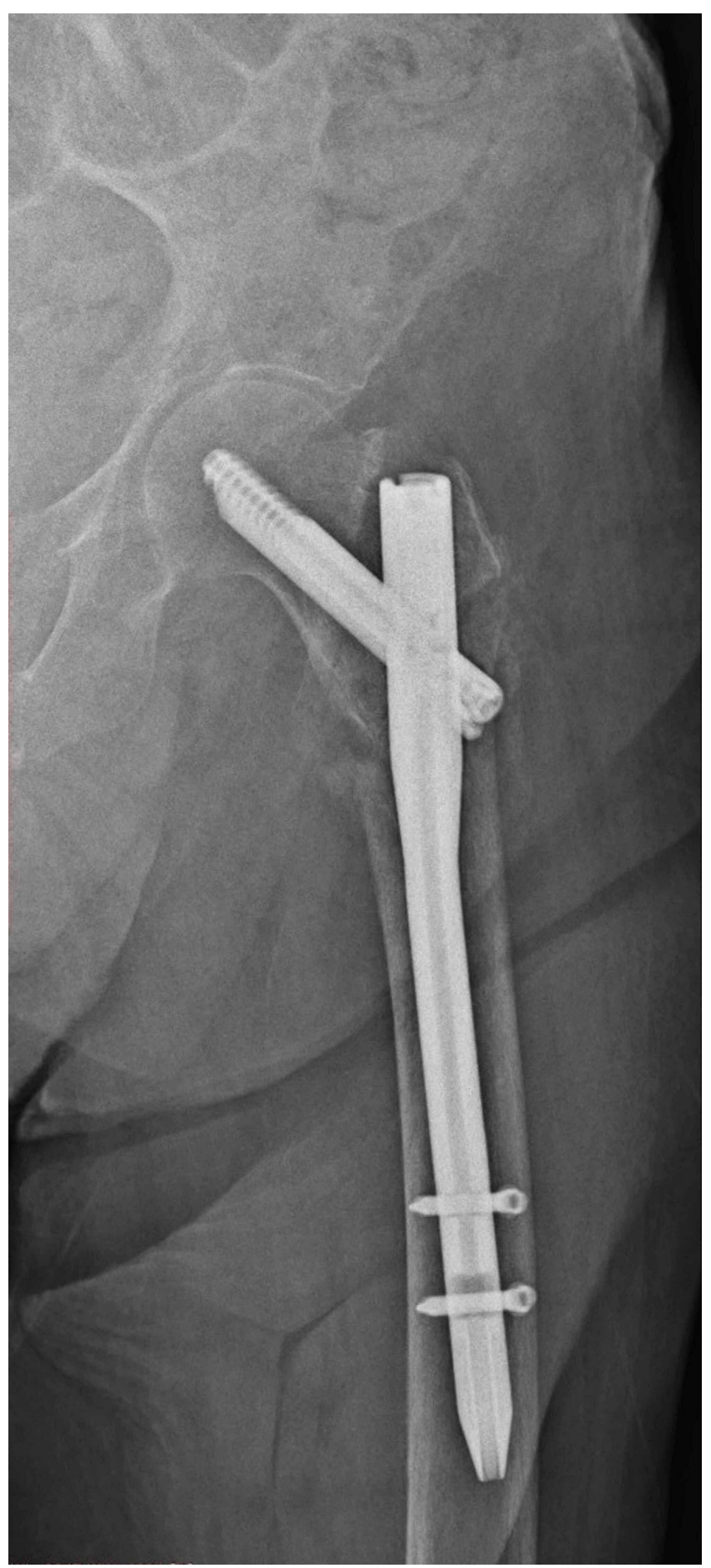

FIGURE 6: Anterior-posterior hip radiography at the third postoperative month of the patient

\begin{tabular}{|c|c|c|c|}
\hline & \multicolumn{2}{|c|}{ Mean \pm SD (median) } & \multirow{2}{*}{$\mathbf{p}$} \\
\hline & $\mathrm{CRH}$ & PFN & \\
\hline Age & $82.2 \pm 5.7$ & $81.5 \pm 7.4$ & ${ }^{\mathrm{a}} 0.504$ \\
\hline The average length of hospital stay (day) & $10.6 \pm 4.1(10)$ & $9.2 \pm 3.1(9)$ & ${ }^{\mathrm{b}} 0.009^{\star *}$ \\
\hline Time from hospitalization to operation (day) & $6 \pm 2.4(5)$ & $5.9 \pm 2.9(5)$ & ${ }^{\mathrm{b}} 0.819$ \\
\hline
\end{tabular}




\section{Cureus}

\begin{tabular}{|c|c|c|c|}
\hline Intraoperative bleeding amount(ml) & $514.6 \pm 63.7$ & $127.3 \pm 42.6$ & ${ }^{\mathrm{a}} 0.001^{\text {** }}$ \\
\hline Harris hip score & $54.5 \pm 18.1$ & $76.2 \pm 16.8$ & ${ }^{\mathrm{a}} 0.001^{\star \star}$ \\
\hline \multirow[t]{2}{*}{ Operation time(min) } & $71.4 \pm 13.3$ & $46.5 \pm 10.7$ & ${ }^{\mathrm{a}} 0.001^{\star *}$ \\
\hline & $\mathrm{n} ; \%$ & $\mathrm{n} ; \%$ & \\
\hline \multicolumn{4}{|l|}{ Gender } \\
\hline Male & $27 ; 28.7$ & $21 ; 27.3$ & ${ }^{\mathrm{c}} 0.834$ \\
\hline Female & $67 ; 71.3$ & $56 ; 72.6$ & \\
\hline \multicolumn{4}{|l|}{ Evans classification } \\
\hline Type 3 & $27 ; 28.7$ & $23 ; 29.9$ & ${ }^{\circ} 0.405$ \\
\hline Type 4 & $52 ; 55.3$ & $47 ; 61$ & \\
\hline Type 5 & $15 ; 16$ & $7 ; 9.1$ & \\
\hline \multicolumn{4}{|l|}{ AO classification } \\
\hline Tyре A2 & 79; 84 & $67 ; 87$ & ${ }^{\circ} 0.584$ \\
\hline Type A3 & $15 ; 16$ & $10 ; 13$ & \\
\hline \multicolumn{4}{|l|}{ Pre-existing disease } \\
\hline No & $11 ; 11.7$ & $5 ; 6.5$ & ${ }^{\mathrm{d}} 0.368$ \\
\hline Yes & $83 ; 88.3$ & 72; 93.5 & \\
\hline \multicolumn{4}{|l|}{ Blood transfusion } \\
\hline No & $30 ; 31.9$ & $61 ; 79.2$ & ${ }^{\mathrm{c}} 0.001^{\star \star}$ \\
\hline 1 unit & $21 ; 22.3$ & $14 ; 18.2$ & \\
\hline 2 unit & $31 ; 33$ & $2 ; 2.6$ & \\
\hline$\geq 3$ unit & $12 ; 12.8$ & $0 ; 0$ & \\
\hline \multicolumn{4}{|l|}{ ASA score } \\
\hline 2 & $15 ; 16$ & $14 ; 18.2$ & ${ }^{\mathrm{c}} 0.824$ \\
\hline 3 & $79 ; 84$ & $63 ; 81.8$ & \\
\hline \multicolumn{4}{|l|}{ Exitus } \\
\hline No & $55 ; 58.5$ & $57 ; 74$ & ${ }^{\circ} 0.034^{*}$ \\
\hline Ex & $39 ; 41.5$ & $20 ; 26$ & \\
\hline \multicolumn{4}{|l|}{ Revision } \\
\hline No & $86 ; 91.5$ & $71 ; 92.2$ & $d_{1.000}$ \\
\hline Yes & 8; 8.5 & $6 ; 7.8$ & \\
\hline \multicolumn{4}{|l|}{ Complication } \\
\hline No & 74; 78.7 & $63 ; 81.8$ & ${ }^{\mathrm{C}} 0.614$ \\
\hline Yes & $20 ; 21.3$ & 14; 18.2 & \\
\hline
\end{tabular}

\section{TABLE 1: Evaluations by groups}

aStudent t-test; ${ }^{\mathrm{b}}$ Mann-Whitney U test; ${ }^{\mathrm{C}}$ Pearson chi-square test; dContinuity correction (Yates) test; ${ }^{*} \mathrm{p}<0.05 ;{ }^{* *} \mathrm{p}<0.01$.

CRH: calcar-replacement hemiarthroplasty, PFN: proximal femoral nail. 
AO fracture classification was type A2 in 79 (84\%) cases and A3 in 15 (16\%) in the CRH group, and A2 in 67 (87\%) cases, and A3 in 10 (13\%) in the PFN group. According to the Evans classification, there were 27 (28.7\%) type 3, 52 (55.3\%) type 4, and 15 (16\%) type 5 fractures in the CRH group. In the PFN group, 23 (29.9\%) patients had type 3, 47 (61\%) had type 4, and 7 (9.1\%) had type 5 fractures. No significant difference was determined between the CRH and PFN groups according to fracture types.

ASA 2 status was determined in 17 patients and ASA 3 in 77 patients in the CRH group, and in the PFN group, 14 patients were ASA 2, and 63 were ASA 3. No significant difference was determined between the groups in respect of the ASA score $(p>0.05)$.

The average time from hospitalization to the operation was six days in the CRH group and 5.3 days in the PFN group. The average length of hospital stay was 10.6 days in the CRH group and 9.2 days in the PFN group (all patients were discharged from the hospital to their homes and did not continue rehabilitation in any hospital). The time from hospitalization to operation and the total length of hospital stay were statistically significantly longer in the CRH group than in the PFN group $(\mathrm{p}<0.01)$ (Table 1$)$.

In the CRH group, 83 patients had pre-existing comorbidities (68 patients had cardiovascular disease, 27 patients had diabetes mellitus, 19 patients had a respiratory disease, and 29 patients had the neurological disease), and 11 patients had no additional disease. In the PFN group, 72 patients had a comorbid disease (cardiovascular disease in 53 patients, diabetes mellitus in 24 patients, respiratory disease in 18 patients, and neurological disease in 24 patients), and 5 patients had no additional disease (Table 2). There was no statistically significant difference between the groups in terms of pre-existing comorbidities $(p>0.05)$.

\begin{tabular}{|c|c|c|}
\hline & \multicolumn{2}{|l|}{$\mathbf{n}$} \\
\hline & CRH & PRN \\
\hline Cardiovascular disease & 68 & 53 \\
\hline Diabetes mellitus & 27 & 24 \\
\hline Respiratory disease & 19 & 18 \\
\hline Neurological disease & 29 & 24 \\
\hline No additional disease & 11 & 5 \\
\hline
\end{tabular}

\section{TABLE 2: Pre-existing comorbidities}

CRH: calcar-replacement hemiarthroplasty, PFN: proximal femoral nail.

When the amount of intraoperative bleeding amount was evaluated (the amount of bleeding was determined by intraoperative gauze count and hemovac drain follow-up), it was determined as $514.6 \mathrm{ml}$ in the CRH group and $127.3 \mathrm{ml}$ in the PFN group. No blood transfusion was required in 30 (31.9\%) patients in the CRH group, 1 unit was transfused in 21 (22.3\%) patients, 2 units in 31 (33\%) patients, and 3 or more units in 12 (12.8\%) patients. In the PFN group, 61 (79.2\%) of the patients did not require a blood transfusion, 1 unit was transfused in $14(18.2 \%)$ patients, and 2 units in $2(2.6 \%)$ patients. No patient in this group required 3 or more units of blood transfusion. When the amount of bleeding and blood transfusion rates of the patients were evaluated, the amount of blood loss in the CRH group was statistically significantly higher than in the PFN group ( $p<0.01)$, and there was a statistically significant difference between the blood transfusion amounts $(p<0.01)$. The rate of no transfusion was higher in the PFN group than in the CRH group, and the rate of transfusion of 2 units or more was higher in the $\mathrm{CRH}$ group.

The mean operating time was 71.4 mins in the CRH group and 46.5 mins in the PFN group. The operating time of the CRH group was found to be statistically significantly longer than that of the PFN group $(\mathrm{p}<0.01)$.

Postoperative complications were determined in 20 (21.3\%) patients in the CRH group (six superficial infections, three deep infections, eight thromboembolic complications, five bedsores), and in 14 (18.2\%) patients in the PFN group (five superficial infections, five thromboembolic complications, four bedsores). No statistically significant difference was determined between the groups in respect of the incidence of postoperative complications $(\mathrm{p}>0.05)$.

When the secondary operation-revision rates of the patients were evaluated, revision surgery was performed in eight (8.5\%) patients in the CRH group (four deep infections, two hip dislocations, two periprosthetic 
fracture) and in six (7.8\%) patients in the PFN group (six cut-outs). There was no statistically significant difference between the revision status according to the groups $(\mathrm{p}>0.05)$.

The two-year survival was evaluated and mortality was seen in 39 (41.5\%) patients in the CRH group and 20 (26\%) patients in the PFN group. The incidence of exitus was significantly higher in the CRH group than in the PFN group $(\mathrm{p}<0.05)$.

In the evaluation of HHS at the final follow-up examination of the patients, the mean HHS was 54.5 in the CRH group and 76.2 in the PFN group. The HHS of the PFN group was statistically significantly higher than that of the CRH group $(\mathrm{p}<0.01)$.

\section{Discussion}

The main findings of the present study are as follows: less total length of hospital stay, better functional outcomes, less surgery-related trauma, and lower mortality rates in the PFN group.

Intertrochanteric femoral fractures account for 3.6\% of all extremity fractures and $45-50 \%$ of all hip fractures in the elderly population [10]. It is very difficult for these elderly patients to return to the pre-injury physical condition due to frequent osteoporosis and delayed fracture healing, which can result in complications and high mortality rates [11]. Treatment of intertrochanteric fractures is surgery, and objective and careful preoperative evaluation of the fracture is required to develop an appropriate treatment plan [12]. Common intertrochanteric fracture treatments include intramedullary fixation (Gamma nail, PFNA), plate fixation (DHS, dynamic condylar screw [DCS]), and BPH. Many elderly patients also have osteoporosis, so fixation may not always be as effective as wished. Most authors currently recommend hemiarthroplasty and PFN as the first surgical option for the treatment of elderly patients with an unstable intertrochanteric fracture $[13,14]$. As a minimally invasive surgery, PFN has good biomechanical properties, making it a preferred method for unstable intertrochanteric fractures associated with osteoporosis [15]. To allow earlier postoperative weight-bearing and to avoid excessive collapse at the fracture site, some surgeons have recommended prosthetic replacement, especially with a calcar-replacement or head and neck-replacement type of prosthesis, for the treatment of unstable intertrochanteric fractures $[1,16]$.

In the present study, similar to the literature, there was no significant difference between the patient classifications of fractures and ASA scores [17,18]. Since the focus in this study was on the results of patients over 65 years of age, these patients are more likely to have an additional disease, and intertrochanteric femoral fractures tend to be unstable. In a retrospective study by Zhou et al. [17], there was determined to be no significant difference between hemiarthroplasty and PFN groups with respect to ASA scores and fracture types. Gormeli et al. also found no difference between ASA scores and fracture types in a retrospective study [18].

In the current study, the average length of hospital stay was 10.6 days in the CRH group, while it was 9.2 days in the PFN group. It has been stated in the literature that the duration of hospitalization of extracapsular hip fractures is similar for both hemiarthroplasty and internal fixation groups [17]. In the present study, the duration of hospitalization in the hemiarthroplasty group was found to be significantly longer than that of the PFN group, and the time from hospitalization to surgery was also significantly longer in the hemiarthroplasty group. The longer hospital stay of the CRH group can be attributed to the higher rate of blood transfusion required for patients in this group and also shorter total hospitalization time could be related to pre-operative hospitalization time.

Since intertrochanteric femoral fractures usually appear at older ages when the bone structure is weakened, the percentage of pre-existing comorbidities in these patients is high. In a study by Jolly et al. [19], it was reported that $64 \%$ of patients in the hemiarthroplasty group and $60 \%$ of patients with PFN had an additional disease. In a retrospective study by Kezmezacar et al. [20], it was similarly stated that there was no statistically significant difference between the patients' comorbidities. In the current study, pre-existing comorbidities were found in $88.3 \%$ of the patients in the CRH group and in $93.5 \%$ of the patients in the PFN group, with no significant difference determined between the groups.

In a randomized prospective study, Kim et al. [21] stated that the duration of surgery, the amount of bleeding, and the amount of blood transfusion were significantly higher in the hemiarthroplasty group than in the PFN group. There was also determined to be no significant difference between the groups in complications seen in the postoperative period. In the present study, the duration of surgery, the amount of bleeding, and the amount of blood transfusion were significantly higher in the hemiarthroplasty group, and there was no significant difference between the groups in respect of complications seen in the postoperative period.

In similar studies in the literature, operation times have been found to be longer in patients with hemiarthroplasty than in the group with PFN $[19,21]$. In the current study, similar to the literature, the operating time of patients who underwent hemiarthroplasty was found to be significantly longer compared to patients who underwent PFN. 
Following intertrochanteric femoral fractures, early complications may occur that do not require a secondary operation such as superficial infection, venous thromboembolism, and bedsores. In their prospective study, Kim et al. [21] encountered early complications in 12 of 29 patients who underwent hemiarthroplasty and in 8 of 29 patients who underwent PFN. Lou et al. [22] also stated in their retrospective study that there was no statistically significant difference between the rates of complications. In the present study, early complications were observed in $21.3 \%$ of patients in the hemiarthroplasty group and in $14 \%$ of patients with PFN, with no significant difference determined between the groups.

Intertrochanteric femur fractures are difficult to reduce intraoperatively and screw loosening and cut out in femoral necks can be often seen with severe osteoporosis. Studies have shown that the use of PFNs in the treatment of intertrochanteric fractures has a failure rate of 7.1-12.5\% [23,24]. In contrast, hemiarthroplasty can quickly restore hip function, so it is mainly used to treat femoral neck fractures in the elderly, including unstable intertrochanteric fractures and failure of intertrochanteric fracture fixation [1]. In the current study, eight (8.5\%) patients in the CRH group and six (7.8\%) patients in the PFN group developed complications requiring revision and there was no statistically significant difference between the two groups.

Kesmezacar et al. [20] compared internal fixation and hemiarthroplasty in elderly patients with pertrochanteric femur fractures and reported that a mortality rate of $48.8 \%$ in the first six months in patients undergoing endoprosthesis, while this rate was $34.2 \%$ in patients with internal fixation. Kim et al. [21] compared the results of hemiarthroplasty and PFN with internal fixation in unstable pertrochanteric fractures and reported mortality as 55\% in the arthroplasty group in the third year, but $17 \%$ in the PFN group. In the present study, the two-year mortality rate was significantly higher in the hemiarthroplasty group than in the PFN group.

In a retrospective study by Gormeli et al. [18], the HHS was found to be higher in the PFN group but there was no significant difference compared to the hemiarthroplasty group. In a prospective study by Jolly et al. [19] comparing hemiarthroplasty and PFN, the HHS at the end of the first postoperative year was better in the PFN group. Similarly, in the present study, the HHS at the end of the first year was found to be significantly higher in the PFN group than in the hemiarthroplasty group.

This study had some limitations, primarily the retrospective design, small patient group, and mid-term follow-up. Long-term analyses are unlikely in an elderly patient population due to the short life expectancy.

\section{Conclusions}

In conclusion, both hemiarthroplasty and PFN produce satisfactory results in surgically treated intertrochanteric femur fractures. Both groups are associated with their own complications, but in the PFN group, better functional results, less surgery-related trauma, and lower mortality rates are the main advantages. Therefore, the clinician should select the ideal method for each patient, but it can be considered that internal fixation may be a more effective, and appropriate treatment method for elderly patients with an extracapsular proximal femur fracture.

\section{Additional Information \\ Disclosures}

Human subjects: Consent was obtained or waived by all participants in this study. Animal subjects: All authors have confirmed that this study did not involve animal subjects or tissue. Conflicts of interest: In compliance with the ICMJE uniform disclosure form, all authors declare the following: Payment/services info: All authors have declared that no financial support was received from any organization for the submitted work. Financial relationships: All authors have declared that they have no financial relationships at present or within the previous three years with any organizations that might have an interest in the submitted work. Other relationships: All authors have declared that there are no other relationships or activities that could appear to have influenced the submitted work.

\section{References}

1. Emami M, Manafi A, Hashemi B, Nemati A, Safari S: Comparison of intertrochanteric fracture fixation with dynamic hip screw and bipolar hemiarthroplasty techniques. Arch Bone Jt Surg. 2013, 1:14-7.

2. Zuckerman JD: Hip fracture. N Engl J Med. 1996, 334:1519-25. 10.1056/NEJM199606063342307

3. Lindskog DM, Baumgaertner MR: Unstable intertrochanteric hip fractures in the elderly . J Am Acad Orthop Surg. 2004, 12:179-90. 10.5435/00124635-200405000-00006

4. Blomfeldt R, Törnkvist H, Eriksson K, Söderqvist A, Ponzer S, Tidermark J: A randomised controlled trial comparing bipolar hemiarthroplasty with total hip replacement for displaced intracapsular fractures of the femoral neck in elderly patients. J Bone Joint Surg Br. 2007, 89:160-5. 10.1302/0301-620X.89B2.18576

5. Röder F, Schwab M, Aleker T, Mörike K, Thon KP, Klotz U: Proximal femur fracture in older patients-rehabilitation and clinical outcome. Age Ageing. 2003, 32:74-80. 10.1093/ageing/32.1.74

6. Fichman SG, Mäkinen TJ, Safir O, Vincent A, Lozano B, Kashigar A, Kuzyk PR: Arthroplasty for unstable pertrochanteric hip fractures may offer a lower re-operation rate as compared to cephalomedullary nailing. 
Int Orthop. 2016, 40:15-20. 10.1007/s00264-015-2794-7

7. Gupta KL: Comparative assessment of primary and secondary outcome with PFNA and hemiarthroplasty for senile intertrochanteric fractures management: a prospective randomized clinical study. Int J Med Biomed Stud. 2020, 4:205-8. 10.32553/ijmbs.v4i3.1091

8. Maru N, Rasik D, Parag T: Unstable intertrochanteric fractures in high risk elderly patients treated with primary bipolar hemiarthroplasty: retrospective case series. Int J Orthop. 2019, 5:616-9. 10.22271/ortho.2019.v5.i4k.1741

9. Harris WH: Traumatic arthritis of the hip after dislocation and acetabular fractures: treatment by mold arthroplasty. An end-result study using a new method of result evaluation. J Bone Joint Surg Am. 1969, 51:737-55.

10. Kokoroghiannis C, Aktselis I, Deligeorgis A, Fragkomichalos E, Papadimas D, Pappadas I: Evolving concepts of stability and intramedullary fixation of intertrochanteric fractures - a review. Injury. 2012, 43:686-93. 10.1016/j.injury.2011.05.031

11. Gaumetou E, Zilber S, Hernigou P: Non-simultaneous bilateral hip fracture: epidemiologic study of 241 hip fractures. Orthop Traumatol Surg Res. 2011, 97:22-7. 10.1016/j.otsr.2010.07.011

12. Han SK, Lee BY, Kim YS, Choi NY: Usefulness of multi-detector CT in Boyd-Griffin type 2 intertrochanteric fractures with clinical correlation. Skeletal Radiol. 2010, 39:543-9. 10.1007/s00256-009-0795-6

13. Setiobudi T, Ng YH, Lim CT, Liang S, Lee K, Das De S: Clinical outcome following treatment of stable and unstable intertrochanteric fractures with dynamic hip screw. Ann Acad Med Singap. 2011, 40:482-7.

14. Li J, Chen JK, Zhou K, Shen B, Ni XM, Chen L: [Application of dynamic hip screw with modified reamer in intertrochanteric fracture in the elderly]. Zhongguo Gu Shang. 2011, 24:362-5.

15. Yang YH, Wang YR, Jiang SD, Jiang LS: Proximal femoral nail antirotation and third-generation Gamma nail: which is a better device for the treatment of intertrochanteric fractures?. Singapore Med J. 2013, 54:446-50. 10.11622/smedj.2013152

16. Haentjens P, Lamraski G: Endoprosthetic replacement of unstable, comminuted intertrochanteric fracture of the femur in the elderly, osteoporotic patient: a review. Disabil Rehabil. 2005, 15:1167-80. 10.1080/09638280500055966

17. Zhou S, Liu J, Zhen P, et al.: Proximal femoral nail anti-rotation versus cementless bipolar hemiarthroplasty for unstable femoral intertrochanteric fracture in the elderly: a retrospective study. BMC Musculoskelet Disord. 2019, 20:500. 10.1186/s12891-019-2793-8

18. Görmeli G, Korkmaz MF, Görmeli CA, Adanaş C, Karataş T, Şimşek SA: Comparison of femur intertrochanteric fracture fixation with hemiarthroplasty and proximal femoral nail systems. Ulus Travma Acil Cerrahi Derg. 2015, 21:503-8. 10.5505/tjtes.2015.96166

19. Jolly A, Bansal R, More AR, Pagadala MB: Comparison of complications and functional results of unstable intertrochanteric fractures of femur treated with proximal femur nails and cemented hemiarthroplasty. J Clin Orthop Trauma. 2019, 10:296-301. 10.1016/j.jcot.2017.09.015

20. Kesmezacar H, Oğüt T, Bilgili MG, Gökay S, Tenekecioğlu Y: [Treatment of intertrochanteric femur fractures in elderly patients: internal fixation or hemiarthroplasty]. Acta Orthop Traumatol Turc. 2005, 39:287-94.

21. Kim SY, Kim YG, Hwang JK: Cementless calcar-replacement hemiarthroplasty compared with intramedullary fixation of unstable intertrochanteric fractures. A prospective, randomized study. J Bone Joint Surg Am. 2005, 87:2186-92. 10.2106/JBJS.D.02768

22. Luo X, He S, Zeng D, Lin L, Li Q: Proximal femoral nail antirotation versus hemiarthroplasty in the treatment of senile intertrochanteric fractures: case report. Int J Surg Case Rep. 2017, 38:37-42. 10.1016/j.ijscr.2017.04.027

23. Papasimos S, Koutsojannis CM, Panagopoulos A, Megas P, Lambiris E: A randomised comparison of AMBI, TGN and PFN for treatment of unstable trochanteric fractures. Arch Orthop Trauma Surg. 2005, 125:462-8. 10.1007/s00402-005-0021-5

24. Boldin C, Seibert FJ, Fankhauser F, Peicha G, Grechenig W, Szyszkowitz R: The proximal femoral nail (PFN) a minimal invasive treatment of unstable proximal femoral fractures: a prospective study of 55 patients with a follow-up of 15 months. Acta Orthop Scand. 2003, 74:53-8. 10.1080/00016470310013662 